\title{
A Description of Pseudorapidity Distributions of Charged Particles Produced in Au+Au Collisions at RHIC Energies
}

\author{
Z. J. Jiang $(D$, Dongfang Xu, and Yan Huang \\ College of Science, University of Shanghai for Science and Technology, Shanghai 200093, China \\ Correspondence should be addressed to Z. J. Jiang; jzj265@163.com
}

Received 15 October 2017; Revised 10 December 2017; Accepted 27 December 2017; Published 24 January 2018

Academic Editor: Fu-Hu Liu

Copyright ( 2018 Z. J. Jiang et al. This is an open access article distributed under the Creative Commons Attribution License, which permits unrestricted use, distribution, and reproduction in any medium, provided the original work is properly cited. The publication of this article was funded by $\mathrm{SCOAP}^{3}$.

\begin{abstract}
In heavy ion collisions, charged particles come from two parts: the hot and dense matter and the leading particles. In this paper, the hot and dense matter is assumed to expand according to the hydrodynamic model including phase transition and decouples into particles via the prescription of Cooper-Frye. The leading particles are as usual supposed to have Gaussian rapidity distributions with the number equaling that of participants. The investigations of this paper show that, unlike low energy situations, the leading particles are essential in describing the pseudorapidity distributions of charged particles produced in high energy heavy ion collisions. This might be due to the different transparencies of nuclei at different energies.
\end{abstract}

\section{Introduction}

The BNL Relativistic Heavy Ion Collider (RHIC) accelerates nuclei up to the center-of-mass energies from a dozen $\mathrm{GeV}$ to $200 \mathrm{GeV}$ per nucleon. In the past decade, the measurements from such collisions have triggered an extensive research for the properties of matter at extreme conditions of very high temperature and energy densities [1-33]. One of the most important achievements from such research is the discovery that the matter created in nucleus-nucleus collisions at RHIC energies is in the state of strongly coupled quark-gluon plasma (sQGP) exhibiting a clear collective behavior nearly like a perfect fluid with very low viscosity [10-33].

The best approach for describing the space-time evolution of fluid-like sQGP is the relativistic hydrodynamics. However, owing to the formidable complexities of hydrodynamic equations, the most analytical work so far is mainly limited to $1+1$ expansion for a perfect fluid with simple equation of state, which can be found as an important application in the analysis of the pseudorapidity distributions of charged particles in high energy physics. In this paper, combining the effects of leading particles, we will discuss such distributions in the framework of $1+1$ hydrodynamic model including phase transition [10].

\section{A Brief Introduction to the Model}

Here, for the purpose of completeness and applications, we will list the key ingredients of the hydrodynamic model [10].

(1) The expansion of fluid is subject to the conservation of energy and momentum. This is reflected in continuity equation

$$
\frac{\partial T^{\mu \nu}}{\partial x^{\nu}}=0, \quad \mu, \nu=0,1,
$$

where $T^{\mu v}$ is the energy-momentum tensor. For a perfect fluid

$$
T^{\mu \nu}=(\varepsilon+p) u^{\mu} u^{\nu}-p g^{\mu \nu},
$$

where

$$
u^{\mu}=\left(u^{0}, u^{1}\right)=\left(\cosh y_{F}, \sinh y_{F}\right), \quad u^{\mu} u_{\mu}=1,
$$

is the 4 -velocity of fluid and $y_{F}$ is its rapidity. $\varepsilon$ and $p$ in (2) are the energy density and pressure of fluid, which meet the thermodynamical relations

$$
\begin{aligned}
\varepsilon+p & =T s, \\
\mathrm{~d} \varepsilon & =T \mathrm{~d} s, \\
\mathrm{~d} p & =s \mathrm{~d} T,
\end{aligned}
$$


where $T$ and $s$ are the temperature and entropy density of fluid, respectively. To close (1), another relation, namely, the equation of state

$$
\frac{\mathrm{d} p}{\mathrm{~d} \varepsilon}=\frac{s \mathrm{~d} T}{T \mathrm{~d} s}=c_{s}^{2}
$$

is needed, where $c_{s}$ is the sound speed of fluid, which takes different values in SQGP and in hadronic phase.

(2) Project (1) to the direction of $u_{\mu}$ and the direction perpendicular to $u_{\mu}$, respectively. This leads to equations

$$
\begin{aligned}
\frac{\partial\left(s u^{\nu}\right)}{\partial x^{\nu}} & =0, \\
\frac{\partial\left(T \sinh y_{F}\right)}{\partial t}+\frac{\partial\left(T \cosh y_{F}\right)}{\partial z} & =0 .
\end{aligned}
$$

Equation (6) is the continuity equation for entropy conservation. Equation (7) means the existence of a scalar function $\phi$ satisfying relations

$$
\begin{aligned}
& \frac{\partial \phi}{\partial t}=T \cosh y_{F} \\
& \frac{\partial \phi}{\partial z}=-T \sinh y_{F} .
\end{aligned}
$$

From $\phi$ and Legendre transformation, Khalatnikov potential $\chi$ is introduced via relation

$$
\chi=\phi-t T \cosh y_{F}+z T \sinh y_{F} .
$$

In terms of $\chi$, the variables $t$ and $z$ can be expressed as

$$
\begin{aligned}
& t=\frac{e^{\theta}}{T_{0}}\left(\frac{\partial \chi}{\partial \theta} \cosh y_{F}+\frac{\partial \chi}{\partial y_{F}} \sinh y_{F}\right), \\
& z=\frac{e^{\theta}}{T_{0}}\left(\frac{\partial \chi}{\partial \theta} \sinh y_{F}+\frac{\partial \chi}{\partial y_{F}} \cosh y_{F}\right),
\end{aligned}
$$

where $T_{0}$ is the initial temperature of fluid and $\theta=\ln \left(T_{0} / T\right)$. Through the above equations, the coordinate base of $(t, z)$ is transformed to that of $\left(\theta, y_{F}\right)$, and (6) is translated into the so-called telegraphy equation

$$
\frac{\partial^{2} \chi}{\partial \theta^{2}}-2 \beta \frac{\partial \chi}{\partial \theta}-\frac{1}{c_{s}^{2}} \frac{\partial^{2} \chi}{\partial y_{F}^{2}}=0, \quad \beta=\frac{1-c_{s}^{2}}{2 c_{s}^{2}}
$$

(3) Along with the expansions of matter created in collisions, it becomes cooler and cooler. As its temperature drops from the initial $T_{0}$ to the critical $T_{c}$, phase transition occurs. The matter transforms from sQGP state to hadronic state. The produced hadrons are initially in the violent and frequent collisions. The major part of these collisions is inelastic. Hence, the abundances of identified hadrons are changing. Furthermore, the mean free paths of these primary hadrons are very short. The movement of them is still like that of a fluid meeting (11) with only difference being the value of $c_{s}$. In sQGP, $c_{s}=c_{0}=1 / \sqrt{3}$, which is the sound speed of a massless perfect fluid, being the maximum of $c_{s}$. In the hadronic state, $c_{s}=c_{h}<c_{0}$. At the point of phase transition, that is, as $T=T_{c}, c_{s}$ is discontinuous.

(4) The solution of (11) for the sector of sQGP is [10]

$$
\chi_{0}\left(\theta, y_{F}\right)=\frac{q_{0} c_{0}}{2} e^{\beta_{0} \theta} I_{0}\left(\beta_{0} c_{0} \sqrt{y_{0}^{2}(\theta)-y_{F}^{2}}\right),
$$

where $q_{0}$ is a constant determined by tuning the theoretical results to experimental data and $I_{0}$ is the 0 th-order modified Bessel function of the first kind, and

$$
\begin{aligned}
\beta_{0} & =\frac{1-c_{0}^{2}}{2 c_{0}^{2}}=1, \\
y_{0}(\theta) & =\frac{\theta}{c_{0}} .
\end{aligned}
$$

In the sector of hadrons, the solution of (11) is [10]

$$
\chi_{h}\left(\theta, y_{F}\right)=\frac{q_{0} c_{0}}{2} B(\theta) I_{0}\left[\lambda\left(\theta, y_{F}\right)\right]
$$

where

$$
\begin{aligned}
B(\theta) & =e^{\beta_{h}\left(\theta-\theta_{c}\right)+\beta_{0} \theta_{c}}, \\
\lambda\left(\theta, y_{F}\right) & =\beta_{h} c_{h} \sqrt{y_{h}^{2}(\theta)-y_{F}^{2}}, \\
\beta_{h} & =\frac{1-c_{h}^{2}}{2 c_{h}^{2}}, \\
y_{h}(\theta) & =\frac{\theta-\theta_{c}}{c_{h}}+\frac{\theta_{c}}{c_{0}}, \\
\theta_{c} & =\ln \left(\frac{T_{0}}{T_{c}}\right) .
\end{aligned}
$$

\section{The Pseudorapidity Distributions of Charged Particles}

(1) The Invariant Multiplicity Distributions of Charged Particles Frozen Out from $s Q G P$. From Khalatnikov potential $\chi$, the rapidity distributions of charged particles frozen out from fluid-like sQGP read [34]

$$
\frac{\mathrm{d} N_{\mathrm{SQGP}}}{\mathrm{d} y_{F}}=\frac{q_{0} c_{0}}{2} A(b)\left(\cosh y \frac{\mathrm{d} z}{\mathrm{~d} y_{F}}-\sinh y \frac{\mathrm{d} t}{\mathrm{~d} y_{F}}\right),
$$

where $A(b)$ is the area of overlap region of collisions, being the function of impact parameter $b$ or centrality cuts. Inserting (10) into the above equation, the part in the round brackets becomes

$$
\begin{aligned}
\cosh y & \frac{\mathrm{d} z}{\mathrm{~d} y_{F}}-\sinh y \frac{\mathrm{d} t}{\mathrm{~d} y_{F}} \\
= & \frac{1}{T} c^{2} \frac{\partial}{\partial \theta}\left(\chi+\frac{\partial \chi}{\partial \theta}\right) \cosh \left(y-y_{F}\right) \\
& -\frac{1}{T} \frac{\partial}{\partial y_{F}}\left(\chi+\frac{\partial \chi}{\partial \theta}\right) \sinh \left(y-y_{F}\right) .
\end{aligned}
$$


With the expansions of hadronic matter, it continues becoming cooler. According to the prescription of CooperFrye [34], as the temperature drops to the freeze-out temperature $T_{\mathrm{FO}}$, the inelastic collisions among hadrons cease. The yields of identified hadrons remain unchanged becoming the measured results in experiments. The invariant multiplicity distributions of charged particles equal $[10,15,34]$

$$
\begin{aligned}
& \frac{\mathrm{d}^{2} N_{\mathrm{sQGP}}}{2 \pi p_{T} \mathrm{~d} y \mathrm{~d} p_{T}}=\frac{1}{(2 \pi)^{3}} \int \frac{\mathrm{d} N_{\mathrm{sQGP}}}{\mathrm{d} y_{F}} \\
& \left.\cdot \frac{m_{T} \cosh \left(y-y_{F}\right)}{\exp \left\{\left[m_{T} \cosh \left(y-y_{F}\right)-\mu_{B}\right] / T\right\}+\delta}\right|_{T=T_{\mathrm{FO}}} \mathrm{d} y_{F},
\end{aligned}
$$

where $m_{T}=\sqrt{m^{2}+p_{T}^{2}}$ is the transverse mass of produced charged particle with rest mass $m . \mu_{B}$ in (18) is the baryochemical potential. For Fermi charged particles, $\delta=1$ in the denominator of (18), and for Bosons, $\delta=-1$. The meaning of (18) is evident. It is the convolution of $\mathrm{d} N_{\mathrm{SQGP}} / \mathrm{d} y_{F}$ with the energy of the charged particles in the state with temperature $T$.

The integral interval of $y_{F}$ in (18) is $\left[-y_{h}\left(\theta_{f}\right), y_{h}\left(\theta_{f}\right)\right]$. The integrand is evaluated with $T=T_{\mathrm{FO}}$. At this moment, the fluid freezes out into the charged particles. Replacing $\chi$ in (17) by $\chi_{h}$ of (14), it becomes

$$
\begin{aligned}
& \left.\left(\cosh y \frac{\mathrm{d} z}{\mathrm{~d} y_{F}}-\sinh y \frac{\mathrm{d} t}{\mathrm{~d} y_{F}}\right)\right|_{T=T_{\mathrm{FO}}}=\frac{1}{T_{\mathrm{FO}}}\left(\beta_{h} c_{h}\right)^{2} \\
& \cdot B\left(\theta_{\mathrm{FO}}\right)\left[S\left(\theta_{\mathrm{FO}}, y_{F}\right) \sinh \left(y-y_{F}\right)\right. \\
& \left.+C\left(\theta_{\mathrm{FO}}, y_{F}\right) \cosh \left(y-y_{F}\right)\right]
\end{aligned}
$$

where

$$
\begin{aligned}
S( & \left.\theta_{\mathrm{FO}}, y_{F}\right) \\
& =\frac{\beta_{h} y_{F}}{\lambda\left(\theta_{\mathrm{FO}}, y_{F}\right)}\left\{\frac{\beta_{h} c_{h} y_{h}\left(\theta_{\mathrm{FO}}\right)}{\lambda\left(\theta_{\mathrm{FO}}, y_{F}\right)} I_{0}\left[\lambda\left(\theta_{\mathrm{FO}}, y_{F}\right)\right]\right. \\
& \left.+\left[\frac{\beta_{h}+1}{\beta_{h}}-\frac{2 \beta_{h} c_{h} y_{h}\left(\theta_{\mathrm{FO}}\right)}{\lambda^{2}\left(\theta_{\mathrm{FO}}, y_{F}\right)}\right] I_{1}\left[\lambda\left(\theta_{\mathrm{FO}}, y_{F}\right)\right]\right\}, \\
C & \left(\theta_{\mathrm{FO}}, y_{F}\right)=\left\{\frac{\beta_{h}+1}{\beta_{h}}+\frac{\left[\beta_{h} c_{h} y_{h}\left(\theta_{\mathrm{FO}}\right)\right]^{2}}{\lambda^{2}\left(\theta_{\mathrm{FO}}, y_{F}\right)}\right\} \\
& \cdot I_{0}\left[\lambda\left(\theta_{\mathrm{FO}}, y_{F}\right)\right]+\frac{1}{\lambda\left(\theta_{\mathrm{FO}}, y_{F}\right)}\left\{\frac{y_{h}\left(\theta_{\mathrm{FO}}\right)}{c_{h}}+1\right. \\
& \left.-\frac{2\left[\beta_{h} c_{h} y_{h}\left(\theta_{\mathrm{FO}}\right)\right]^{2}}{\lambda^{2}\left(\theta_{\mathrm{FO}}, y_{F}\right)}\right\} I_{1}\left[\lambda\left(\theta_{\mathrm{FO}}, y_{F}\right)\right],
\end{aligned}
$$

where $I_{1}$ is the 1st-order modified Bessel function of the first kind.

(2) The Invariant Multiplicity Distributions of Leading Particles. Investigations have shown that the leading particles are formed outside the overlap region of collisions $[35,36]$. The generation and movement of them are therefore beyond the scope of hydrodynamic description and should be treated separately.

In our previous work [24-26], we once argued that the rapidity distributions of leading particles take the Gaussian form

$$
\begin{aligned}
& \frac{\mathrm{d} N_{\text {Lead }}\left(b, \sqrt{s_{\mathrm{NN}}}, y\right)}{\mathrm{d} y} \\
& =\frac{N_{\text {Lead }}\left(b, \sqrt{s_{\mathrm{NN}}}\right)}{\sqrt{2 \pi} \sigma} \exp \left\{-\frac{\left[|y|-y_{0}\left(b, \sqrt{s_{\mathrm{NN}}}\right)\right]^{2}}{2 \sigma^{2}}\right\},
\end{aligned}
$$

where $y_{0}\left(b, \sqrt{s_{\mathrm{NN}}}\right)$ and $\sigma$ are, respectively, the central position and width of distributions. It can be expected that $y_{0}\left(b, \sqrt{s_{\mathrm{NN}}}\right)$ should increase with increasing energies and centrality cuts, while $\sigma$ should not, at least not apparently, depend on the energies, centrality cuts, and even colliding systems. The specific values of them can be determined by comparing the theoretical results with experimental data. $N_{\text {Lead }}\left(b, \sqrt{s_{\mathrm{NN}}}\right)$ in (21) represents the number of leading particles, which, for an identical nucleus-nucleus collision, equals half of the number of participants.

The investigations have shown that [37], for certain rapidity, the invariant multiplicity distributions of leading particles possess the form

$$
\frac{\mathrm{d}^{2} N_{\text {Lead }}}{2 \pi p_{T} \mathrm{~d} y \mathrm{~d} p_{T}} \propto \exp \left(-a p_{T}^{2}\right),
$$

where $a$ is a constant. Then, as a function of rapidity, the invariant multiplicity distributions of leading particles can be written as

$$
\frac{\mathrm{d}^{2} N_{\text {Lead }}}{2 \pi p_{T} \mathrm{~d} y \mathrm{~d} p_{T}}=\frac{\mathrm{d} N_{\text {Lead }}}{\mathrm{d} y} \frac{a}{\pi} \exp \left(-a p_{T}^{2}\right),
$$

which is normalized to $N_{\text {Lead }}$.

(3) The Pseudorapidity Distributions of Charged Particles. Writing invariant multiplicity distributions in terms of pseudorapidity, we have

$$
\frac{\mathrm{d}^{2} N}{2 \pi p_{T} \mathrm{~d} \eta \mathrm{d} p_{T}}=\sqrt{1-\frac{m^{2}}{m_{T}^{2} \cosh ^{2} y}} \frac{\mathrm{d}^{2} N}{2 \pi p_{T} \mathrm{~d} y \mathrm{~d} p_{T}},
$$

where

$$
\frac{\mathrm{d}^{2} N}{2 \pi p_{T} \mathrm{~d} y \mathrm{~d} p_{T}}=\frac{\mathrm{d}^{2} N_{\text {sQGP }}}{2 \pi p_{T} \mathrm{~d} y \mathrm{~d} p_{T}}+\frac{\mathrm{d}^{2} N_{\text {Lead }}}{2 \pi p_{T} \mathrm{~d} y \mathrm{~d} p_{T}} .
$$

To fulfill the transformation of (24), another relation

$$
y=\frac{1}{2} \ln \left[\frac{\sqrt{p_{T}^{2} \cosh ^{2} \eta+m^{2}}+p_{T} \sinh \eta}{\sqrt{p_{T}^{2} \cosh ^{2} \eta+m^{2}}-p_{T} \sinh \eta}\right]
$$

is in order.

Substituting (18) and (23) into (24) and carrying out the integration of $p_{T}$, we can get the pseudorapidity distributions 


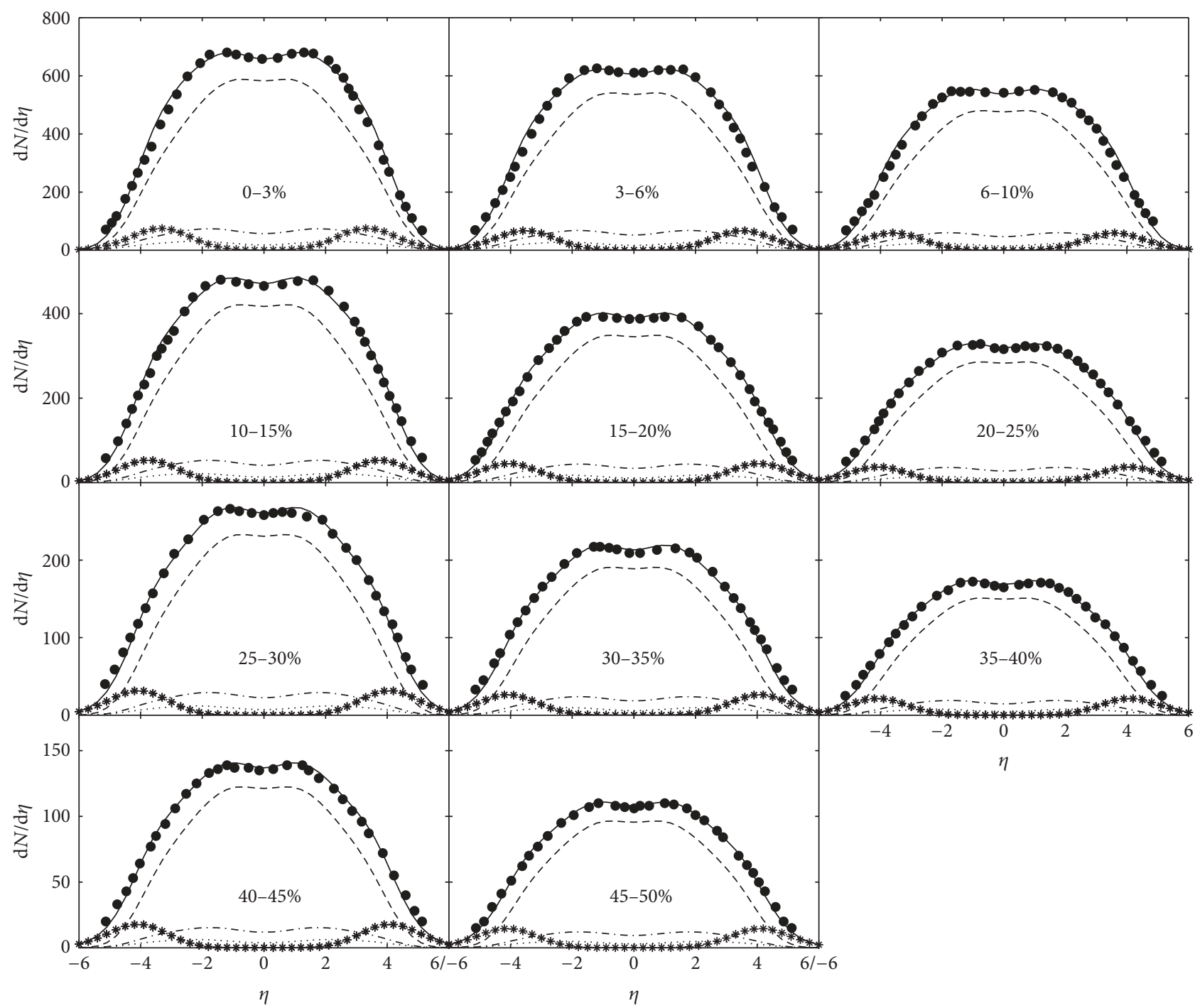

Figure 1: The pseudorapidity distributions of charged particles produced in different centrality Au+Au collisions at $\sqrt{\mathcal{S}_{\mathrm{NN}}}=200 \mathrm{GeV}$. The solid dots are the experimental measurements [5]. The dashed, dashed-dotted, and dotted curves are, respectively, the contribution from pions, kaons, and protons got from the hydrodynamic result of (18). The dotted-star curves are the components of leading particles obtained from (23). The solid curves are the sums of the four types of curves.

of charged particles produced in high energy heavy ion collisions. Figures 1 and 2 show such distributions in $\mathrm{Au}+\mathrm{Au}$ collisions at $\sqrt{s_{\mathrm{NN}}}=200$ and $62.4 \mathrm{GeV}$, respectively. The solid dots in the figures are the experimental measurements [5]. The dashed, dashed-dotted, and dotted curves are, respectively, the contribution from pions, kaons, and protons got from the hydrodynamic result of (18). The dotted-star curves are the components of leading particles obtained from (23). The solid curves are the sums of the four types of curves. $\chi^{2} / \mathrm{NDF}$ for each curve is listed in Table 1 . It can be seen that the combined contribution from both hydrodynamics and leading particles matches up well with experimental data.

Experiments have shown that the overwhelming majority of charged particles produced in $\mathrm{Au}+\mathrm{Au}$ collisions at $\sqrt{s_{\mathrm{NN}}}=200 \mathrm{GeV}$ consists of pions, kaons, and protons with proportions of about $84 \%, 12 \%$, and $4 \%$, respectively [38], which are roughly independent of energies, centrality cuts, and colliding systems. In calculations, the ratios of these three kinds of particles take about the same as these values. $c_{h}$ in (15) takes the values of $c_{h}=0.45$ and 0.42 for $\sqrt{s_{\mathrm{NN}}}=200$ and $62.4 \mathrm{GeV}$ from the investigations of $[15,39-41] . T_{c}$ in (15) takes the well-recognized value of $T_{c}=180 \mathrm{MeV}$. The freezeout temperature $T_{\mathrm{FO}}$ takes the values of $T_{\mathrm{FO}}=120 \mathrm{MeV}$ from the studies of [6], which also shows that the baryochemical potential $\mu_{B}$ in (18) is about equal to 20 and $50 \mathrm{MeV}$ for $\sqrt{s_{\mathrm{NN}}}=200$ and $62.4 \mathrm{GeV}$, respectively. For the most central collisions at these two different energies, $T_{0}$ in (15) takes the values of $T_{0}=0.95$ and $0.68 \mathrm{GeV}$ referring to those given in [15]. This allows us to determine the constant $q_{0}$ in (16) to be $q_{0}=7.38 \times 10^{-4}, 6.01 \times 10^{-4}$, and $4.50 \times 10^{-3}$ for pions, kaons, and protons, respectively. Keeping $q_{0}$ unchanged, $T_{0}$ is fixed for the rest centrality cuts by making theoretical results fit in with experimental data. The results are listed in Table 1. It can be seen that $T_{0}$ decreases slowly with increasing centralities 


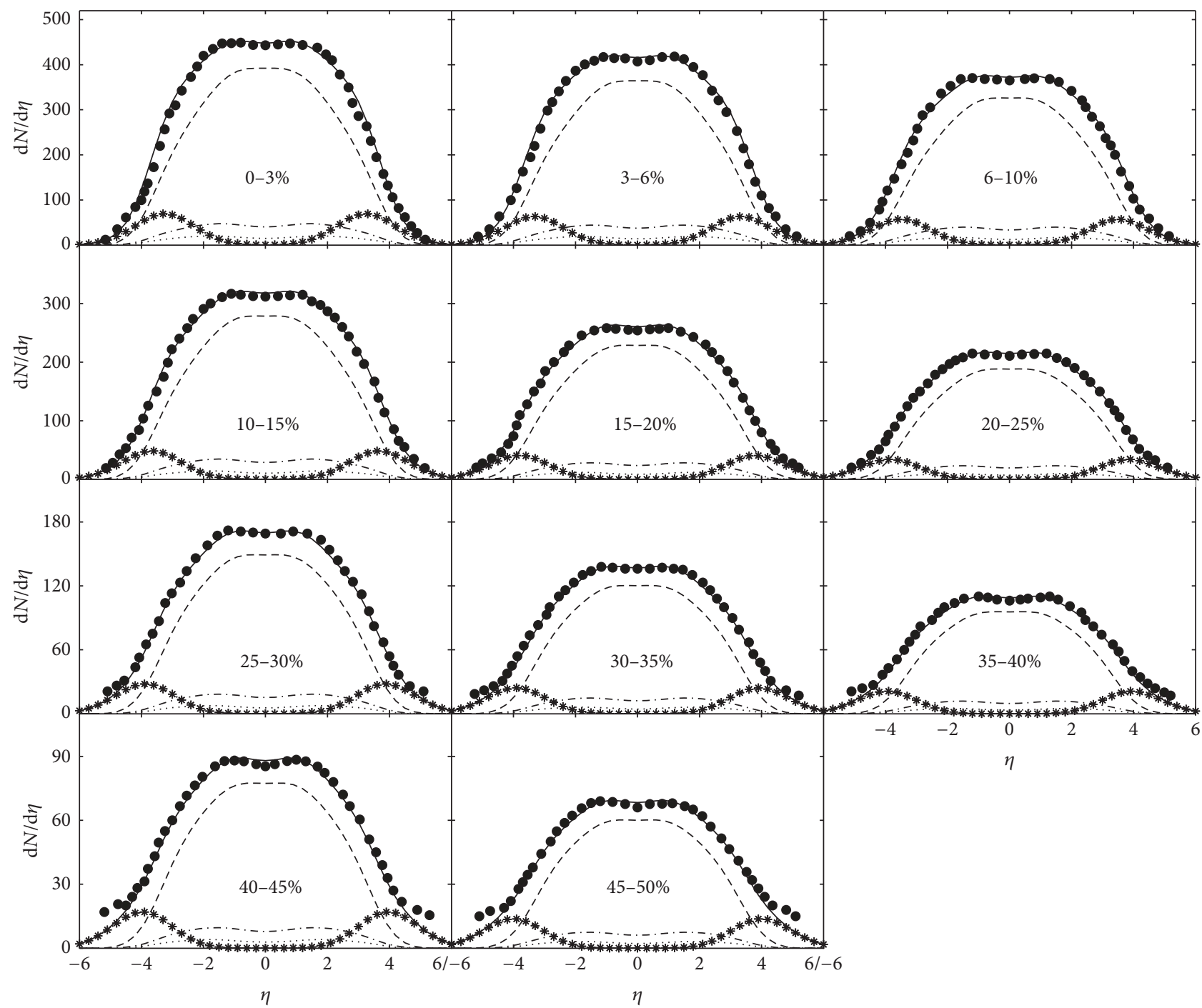

FIGURE 2: The pseudorapidity distributions of charged particles produced in different centrality Au+Au collisions at $\sqrt{s_{\mathrm{NN}}}=62.4 \mathrm{GeV}$. The solid dots are the experimental measurements [5]. The dashed, dashed-dotted, and dotted curves are, respectively, the contribution from pions, kaons, and protons got from the hydrodynamic result of (18). The dotted-star curves are the components of leading particles obtained from (23). The solid curves are the sums of the four types of curves.

TABLE 1: $\chi^{2} / \mathrm{NDF}$, initial temperature $T_{0}$, and central position $y_{0}$ in different centrality Au+Au collisions at $\sqrt{s_{\mathrm{NN}}}=200,62.4$, and $19.6 \mathrm{GeV}$, respectively.

\begin{tabular}{lccccccccccc}
\hline Centrality cuts (\%) & $0-3$ & $3-6$ & $6-10$ & $10-15$ & $15-20$ & $20-25$ & $25-30$ & $30-35$ & $35-40$ & $40-45$ & $45-50$ \\
\hline$\chi^{2} / \mathrm{NDF}$ & & & & & & & & & & & \\
$\quad 200 \mathrm{GeV}$ & 0.496 & 0.693 & 0.402 & 0.260 & 0.310 & 0.217 & 0.354 & 0.151 & 0.161 & 0.123 & 0.089 \\
$62.4 \mathrm{GeV}$ & 0.696 & 0.519 & 0.300 & 0.192 & 0.188 & 0.079 & 0.266 & 0.376 & 0.444 & 0.481 & 0.405 \\
$19.6 \mathrm{GeV}$ & 0.816 & 0.533 & 0.359 & 0.614 & 0.802 & 0.309 & 0.559 & 0.496 & 0.229 & 0.577 & - \\
\hline$T_{0}(\mathrm{GeV})$ & & & & & & & & & & \\
$200 \mathrm{GeV}$ & 0.950 & 0.949 & 0.948 & 0.947 & 0.945 & 0.941 & 0.922 & 0.909 & 0.885 & 0.874 & 0.860 \\
$62.4 \mathrm{GeV}$ & 0.680 & 0.676 & 0.675 & 0.671 & 0.660 & 0.650 & 0.623 & 0.607 & 0.599 & 0.593 & 0.585 \\
$19.6 \mathrm{GeV}$ & 0.551 & 0.549 & 0.547 & 0.543 & 0.539 & 0.533 & 0.529 & 0.518 & 0.510 & 0.493 & - \\
\hline$y_{0}$ & & & & & & & & & & \\
$200 \mathrm{GeV}$ & 2.86 & 3.03 & 3.13 & 3.23 & 3.46 & 3.54 & 3.55 & 3.57 & 3.58 & 3.59 & 3.60 \\
$62.4 \mathrm{GeV}$ & 2.80 & 2.88 & 3.05 & 3.21 & 3.35 & 3.39 & 3.42 & 3.46 & 3.50 & 3.51 & 3.54 \\
\hline
\end{tabular}




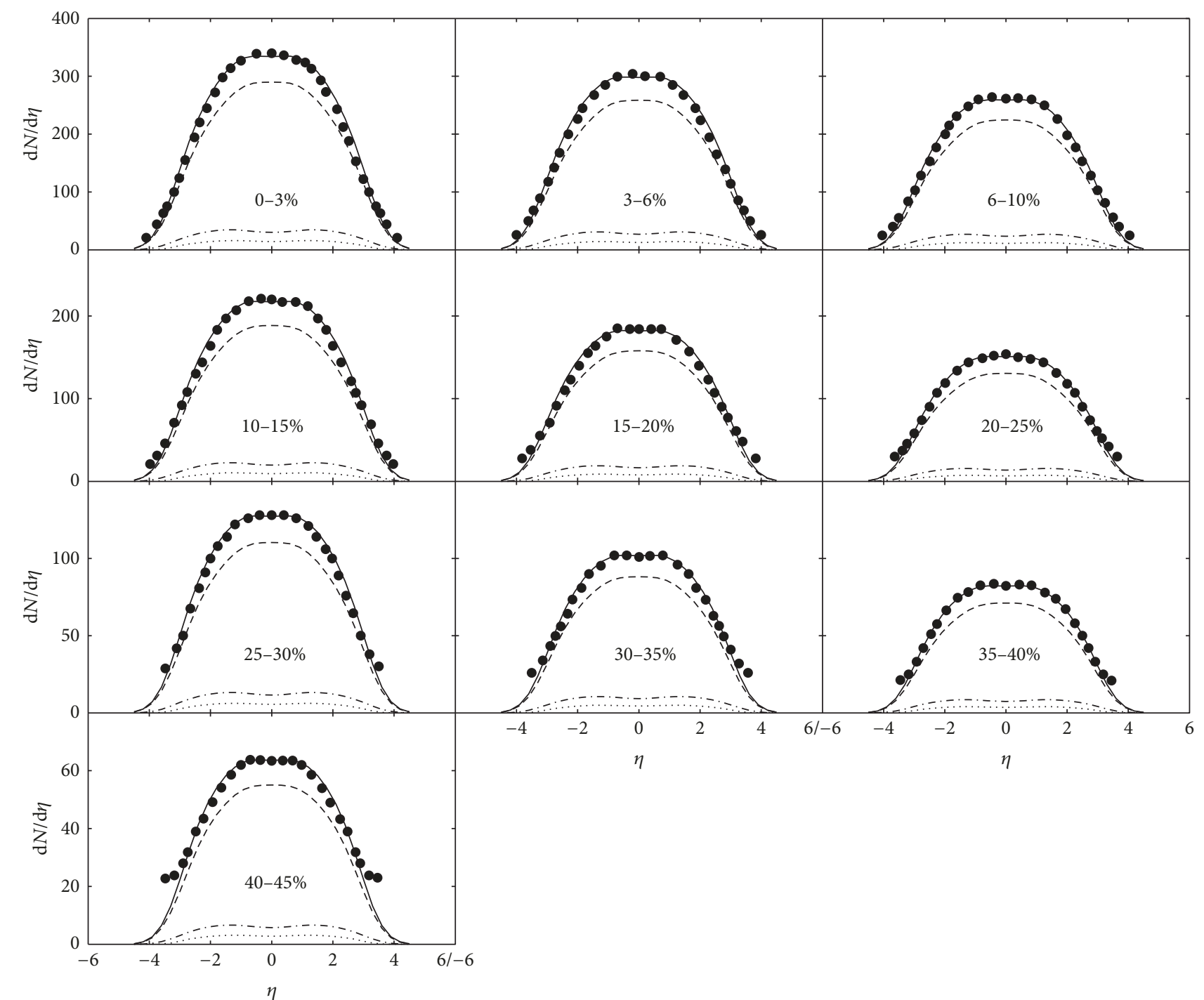

Figure 3: The pseudorapidity distributions of charged particles produced in different centrality Au+Au collisions at $\sqrt{s_{\mathrm{NN}}}=19.6 \mathrm{GeV}$. The solid dots are the experimental measurements [5]. The dashed, dashed-dotted, and dotted curves are, respectively, the contribution from pions, kaons, and protons got from the hydrodynamic results of (18). The solid curves are the sums of the three types of curves.

especially in the first four cuts. Table 1 also lists the central position $y_{0}$ in (21). As addressed above, it increases with increasing energies and centralities. The width parameter $\sigma$ in (21) takes the value of a constant of $\sigma=0.90$, being independent of energies and centrality cuts. The parameter $a$ in (23) takes the value of $a=0.92$ for the two different energies.

Figure 3 shows the pseudorapidity distributions of charged particles produced in $\mathrm{Au}+\mathrm{Au}$ collisions at $\sqrt{s_{\mathrm{NN}}}=$ 19.6 GeV. $\chi^{2} / \mathrm{NDF}$ for each curve is listed in Table 1 . The meanings of different types of curves are the same as those in Figures 1 and 2 . It can be seen that, in the absence of leading particles, the hydrodynamics alone can give a good description to the experimental observations. This is different from Figures 1 and 2, where leading particles are essential in fitting experimental data. This difference might be caused by the different transparencies of nuclei in different energies. As the analyses given in [42], in central $\mathrm{Au}+\mathrm{Au}$ collisions at $\sqrt{s_{\mathrm{NN}}}=$ $200 \mathrm{GeV}$, the leading particles are located at about $y_{0}=2.91$. This position is far away from the mid-rapidity region, where, relative to the low yields of charged particles frozen out from sQGP, the effect of leading particles is evident which should be considered separately. On the contrary, in case of $\mathrm{Au}+\mathrm{Au}$ collisions at $\sqrt{s_{\mathrm{NN}}}=19.6 \mathrm{GeV}, y_{0}=1.28$. This position is so close to the mid-rapidity region that the effect of leading particles is hidden by the large yields of charged particles generated from the freeze-out of sQGP. Therefore, there is no need to consider the contribution of leading particles separately.

In drawing Figure 3, $T_{0}$ takes the values as those listed in Table 1. $c_{h}=0.40$ and $\mu_{B}=210 \mathrm{MeV}$. The other parameters, 
such as $T_{c}, T_{\mathrm{FO}}$, and $q_{0}$, are the same as those used in drawing Figures 1 and 2 .

\section{Conclusions}

By taking into consideration the effect of leading particles, the hydrodynamic model incorporating the phase transition is used to analyze the pseudorapidity distributions of charged particles produced in $\mathrm{Au}+\mathrm{Au}$ collisions at RHIC energies.

The hydrodynamic model contains rich information about transport coefficients of sQGP, such as the sound speed $c_{0}$ in sQGP, the sound speed $c_{h}$ in hadronic phase, the phase transition temperature $T_{c}$, the chemical freeze-out temperature $T_{\mathrm{FO}}$, the baryochemical potential $\mu_{B}$, and the initial temperature $T_{0}$. With the exception of $T_{0}$, the other five coefficients take the values either from the well-known theoretical results or from experimental measurements. As for $T_{0}$, there are no widely accepted results so far. In our calculations, $T_{0}$ in the most central $\mathrm{Au}+\mathrm{Au}$ collisions at $\sqrt{s_{\mathrm{NN}}}=200$ and $62.4 \mathrm{GeV}$ takes the value referring to those given by other investigations, which enables us to ascertain the constant $q_{0}$ in (16). In the rest centrality cuts and in $\mathrm{Au}+\mathrm{Au}$ collisions at $\sqrt{s_{\mathrm{NN}}}=19.6 \mathrm{GeV}, T_{0}$ is determined by maintaining $q_{0}$ unchanged and comparing the theoretical results with experimental data.

The leading particles, by conventional definition, are the particles carrying on the quantum numbers of colliding nucleons and taking away the most part of incident energy. They are separately in projectile and target fragmentation region. The present investigations show that the importance of leading particles in describing the pseudorapidity distributions of charged particles produced in heavy ion collisions is related to the incident energy. At high energy, owing to the high transparency of nuclei, the contribution of leading particles is evident and indispensable, while, at low energy, as a result of poor transparency of nuclei the effect of leading particles is integrated with the results of freeze-out of sQCD. It does not need to be dealt with separately.

\section{Conflicts of Interest}

The authors declare that there are no conflicts of interest regarding the publication of this paper.

\section{Acknowledgments}

This work is supported by the Shanghai Key Lab of Modern Optical System.

\section{References}

[1] C. Adler and STAR Collaboration, "Measurement of inclusive antiprotons from $\mathrm{Au}+\mathrm{Au}$ collisions at $\sqrt{s_{\mathrm{NN}}}=130 \mathrm{GeV}$," Physical Review Letters, vol. 87, Article ID 262302, 2001.

[2] K. Adcox and PHENIX Collaboration, "Centrality dependence of $\pi^{+/-}, K^{+/-}, p$, and $\bar{p}$ production from $\sqrt{s_{\mathrm{NN}}}=130 \mathrm{GeV}$ $\mathrm{Au}+\mathrm{Au}$ collisions at RHIC," Physical Review Letters, vol. 88, Article ID 242301, 2002.
[3] S. S. Adler and PHENIX collaboration, "Elliptic flow of identified hadrons in Au+Au collisions at $\sqrt{s_{\mathrm{NN}}}=200 \mathrm{GeV}$," Physical Review Letters, vol. 91, Article ID 182301, 2003.

[4] K. Adcox and PHENIX Collaboration, "Single identified hadron spectra from $\sqrt{s_{\mathrm{NN}}}=130 \mathrm{GeV} \mathrm{Au}+\mathrm{Au}$ collisions," Physical Review C, vol. 69, Article ID 024904, 2004.

[5] B. Alver and PHOBOS Collaboration, "Charged-particle multiplicity and pseudorapidity distributions measured with the $\mathrm{PHOBOS}$ detector in $\mathrm{Au}+\mathrm{Au}, \mathrm{Cu}+\mathrm{Cu}, d+\mathrm{Au}$, and $p+p$ collisions at ultrarelativistic energies," Physical Review C, vol. 83, Article ID 024913, 2011.

[6] B. I. Abelev and STAR Collaboration, "Systematic measurements of identified particle spectra in $p+p, d+\mathrm{Au}$, and $\mathrm{Au}+\mathrm{Au}$ collisions at the STAR detector," Physical Review C, vol. 79, Article ID 034909, 2009.

[7] A. Adare and PHENIX Collaboration, "Spectra and ratios of identified particles in Au+Au and d+Au collisions at $\sqrt{s_{\mathrm{NN}}}=200$ GeV," Physical Review C, vol. 88, Article ID 024906, 2013.

[8] L. C. Arsene and BRAHMS collaboration, "Rapidity and centrality dependence of particle production for identified hadrons in $\mathrm{Cu}+\mathrm{Cu}$ collisions at $\sqrt{s_{\mathrm{NN}}}=200 \mathrm{GeV}$," Physical Review C, vol. 94, Article ID 014907, 2016.

[9] L. Adamczyk and STAR Collaboration, "Centrality dependence of identified particle elliptic flow in relativistic heavy ion collisions at $\sqrt{s_{\mathrm{NN}}}=7.7-62.4 \mathrm{GeV}$,' Physical Review C, vol. 93, Article ID 014907, 2016.

[10] N. Suzuki, "One-dimensional hydrodynamical model including phase transition," Physical Review C nuclear physics, vol. 81, no. 4, Article ID 044911, 2010.

[11] A. Bialas, R. A. Janik, and R. Peschanski, "Unified description of Bjorken and Landau 1+1 hydrodynamics," Physical Review C nuclear physics, vol. 76, no. 5, Article ID 054901, 2007.

[12] G. Beuf, R. Peschanski, and E. N. Saridakis, "Entropy flow of a perfect fluid in $(1+1)$ hydrodynamics," Physical Review C: Nuclear Physics, vol. 78, no. 6, Article ID 064909, 2008.

[13] T. Csörgo, M. I. Nagy, and M. Csanád, "New family of simple solutions of relativistic perfect fluid hydrodynamics," Physics Letters B, vol. 663, no. 4, pp. 306-311, 2008.

[14] M. Csanád, M. I. Nagy, and S. Lökös, "Exact solutions of relativistic perfect fluid hydrodynamics for a QCD Equation of State," The European Physical Journal A, vol. 48, no. 11, pp. 173178, 2012.

[15] T. Mizoguchi, H. Miyazawa, and M. Biyajima, "A potential including the Heaviside function in the $1+1$ dimensional hydrodynamics by Landau: IIts basic properties and application to data at RHIC energies," The European Physical Journal A, vol. 40, no. 1, pp. 99-108, 2009.

[16] T. R. Taylor and C. Vafa, "Analytic solution for relativistic transverse flow at the softest point," Physics Letters B, vol. 474, no. 1-2, pp. 21-26, 2000.

[17] T. S. Bíró, "Generating new solutions for relativistic transverse flow at the softest point," Physics Letters B, vol. 487, no. 1-2, pp. 133-139, 2000.

[18] C.-Y. Wong, "Landau hydrodynamics reexamined," Physical Review C: Nuclear Physics, vol. 78, no. 5, Article ID 054902, 2008.

[19] A. Bialas and R. Peschanski, "Asymmetric (1+1)-dimensional hydrodynamics in high-energy collisions," Physical Review C: Nuclear Physics, vol. 83, no. 5, Article ID 054905, 2011.

[20] E. K. G. Sarkisyan and A. S. Sakharov, "Relating multihadron production in hadronic and nuclear collisions," The European Physical Journal C, vol. 70, no. 3, pp. 533-541, 2010. 
[21] C. Gale, S. Jeon, and B. Schenke, "Hydrodynamic modeling of heavy-ion collisions," International Journal of Modern Physics A, vol. 28, no. 11, Article ID 1340011, 2013.

[22] U. Heinz and R. Snellings, "Collective flow and viscosity in relativistic heavy-ion collisions," Annual Review of Nuclear and Particle Science, vol. 63, pp. 123-151, 2013.

[23] A. N. Mishra, R. Sahoo, E. K. G. Sarkisyan, and A. S. Sakharov, "Effective-energy budget in multiparticle production in nuclear collisions," The European Physical Journal C, vol. 74, article 3147, 2014.

[24] Z. J. Jiang, Q. G. Li, and H. L. Zhang, "Revised Landau hydrodynamic model and the pseudorapidity distributions of charged particles produced in nucleus-nucleus collisions at maximum energy at the BNL Relativistic Heavy Ion Collider," Physical Review C: Nuclear Physics, vol. 87, no. 4, Article ID 044902, 2013.

[25] Z. J. Jiang, Y. Zhang, H. L. Zhang, and H. P. Deng, "A description of the pseudorapidity distributions in heavy ion collisions at RHIC and LHC energies," Nuclear Physics A, vol. 941, pp. 188200, 2015.

[26] Z. W. Wang, Z. J. Jiang, and Y. S. Zhang, “The investigations of pseudorapidity distributions of final multiplicity in $\mathrm{Au}+\mathrm{Au}$ collisions at high energy," Journal of University of Shanghai for Science and Technology, vol. 31, pp. 322-326, 2009.

[27] J. Qian and U. Heinz, "Hydrodynamic flow amplitude correlations in event-by-event fluctuating heavy-ion collisions," Physical Review C: Nuclear Physics, vol. 94, no. 2, Article ID 024910, 2016.

[28] E. K. G. Sarkisyan, A. N. Mishra, R. Sahoo, and A. S. Sakharov, "Multihadron production dynamics exploring the energy balance in hadronic and nuclear collisions," Physical Review D: Particles, Fields, Gravitation and Cosmology, vol. 93, no. 7, Article ID 079904, 2016.

[29] E. K. G. Sarkisyan, A. N. Mishra, R. Sahoo, and A. S. Sakharov, "Centrality dependence of midrapidity density from $\mathrm{GeV}$ to $\mathrm{TeV}$ heavy-ion collisions in the effective-energy universality picture of hadroproduction," Physical Review D: Particles, Fields, Gravitation and Cosmology, vol. 94, no. 1, Article ID 011501, 2016.

[30] J. Noronha-Hostler, M. Luzum, and J.-Y. Ollitrault, "Hydrodynamic predictions for $5.02 \mathrm{TeV} \mathrm{Pb}-\mathrm{Pb}$ collisions," Physical Review C nuclear physics, vol. 93, no. 3, Article ID 034912, 2016.

[31] H. Niemi, K. J. Eskola, and R. Paatelainen, "Event-by-event fluctuations in a perturbative QCD + saturation + hydrodynamics model: Determining QCD matter shear viscosity in ultrarelativistic heavy-ion collisions," Physical Review C: Nuclear Physics, vol. 93, no. 2, Article ID 024907, 2016.

[32] F. G. Gardim, F. Grassi, M. Luzum, and J. Noronha-Hostler, "Hydrodynamic predictions for mixed harmonic correlations in $200 \mathrm{GeV}$ Au+Au collisions," Physical Review C: Nuclear Physics, vol. 95, no. 3, Article ID 034901, 2017.

[33] M. Alqahtani, M. Nopoush, and M. Strickland, "Quasiparticle anisotropic hydrodynamics for central collisions," Physical Review C: Nuclear Physics, vol. 95, no. 3, Article ID 034906, 2017.

[34] F. Cooper and G. Frye, "Landau's hydrodynamic model of particle production and electron-positron annihilation into hadrons," Physical Review D: Particles, Fields, Gravitation and Cosmology, vol. 11, pp. 192-213, 1975.

[35] A. Berera, M. Strikman, W. Toothacker, W. Walker, and J. Whitmore, "The limiting curve of leading particles from hadronnucleus collisions at infinite A," Physics Letters B, vol. 403, no. 1-2, pp. 1-7, 1997.
[36] J. J. Ryan, "Proceedings of the Annual Meeting of the Division of Particles and Fields of the APS," World Scientific, Singapore, 1993.

[37] A. E. Brenner, D. C. Carey, J. E. Elias et al., "Experimental study of single-particle inclusive hadron scattering and associated multiplicities," Physical Review D: Particles, Fields, Gravitation and Cosmology, vol. 26, no. 7, pp. 1497-1553, 1982.

[38] S. S. Adler and PHENIX collaboration, "Identified charged particle spectra and yields in $\mathrm{Au}+\mathrm{Au}$ collisions at $\sqrt{\boldsymbol{s}_{\mathrm{NN}}}=$ 200 GeV," Physical Review C, vol. 69, Article ID 034909, 2004.

[39] A. Adare and PHENIX Collaboration, "Scaling properties of azimuthal anisotropy in $\mathrm{Au}+\mathrm{Au}$ and $\mathrm{Cu}+\mathrm{Cu}$ collisions at $\sqrt{s_{\mathrm{NN}}}$ $=200$ GeV," Physical Review Letters, vol. 98, Article ID 162301, 2007.

[40] L. N. Gao, Y. H. Chen, H. R. Wei, and F. H. Liu, "Speed of sound parameter from RHIC and LHC heavy-ion data," Advances in High Energy Physics, vol. 2013, Article ID 450247, 8 pages, 2013.

[41] S. Borsányi, G. Endrodi, Z. Fodor et al., “The QCD equation of state with dynamical quarks," Journal of High Energy Physics, vol. 2010, no. 11, article 77, pp. 1-31, 2010.

[42] Z.-J. Jiang, H.-L. Zhang, J. Wang, K. Ma, and L.-M. Cai, “The evolution-dominated hydrodynamics and the pseudorapidity distributions in nucleus-nucleus collisions at low energies at the BNL relativistic heavy ion collider," Chinese Journal of Physics, vol. 52, no. 6, pp. 1676-1685, 2014. 

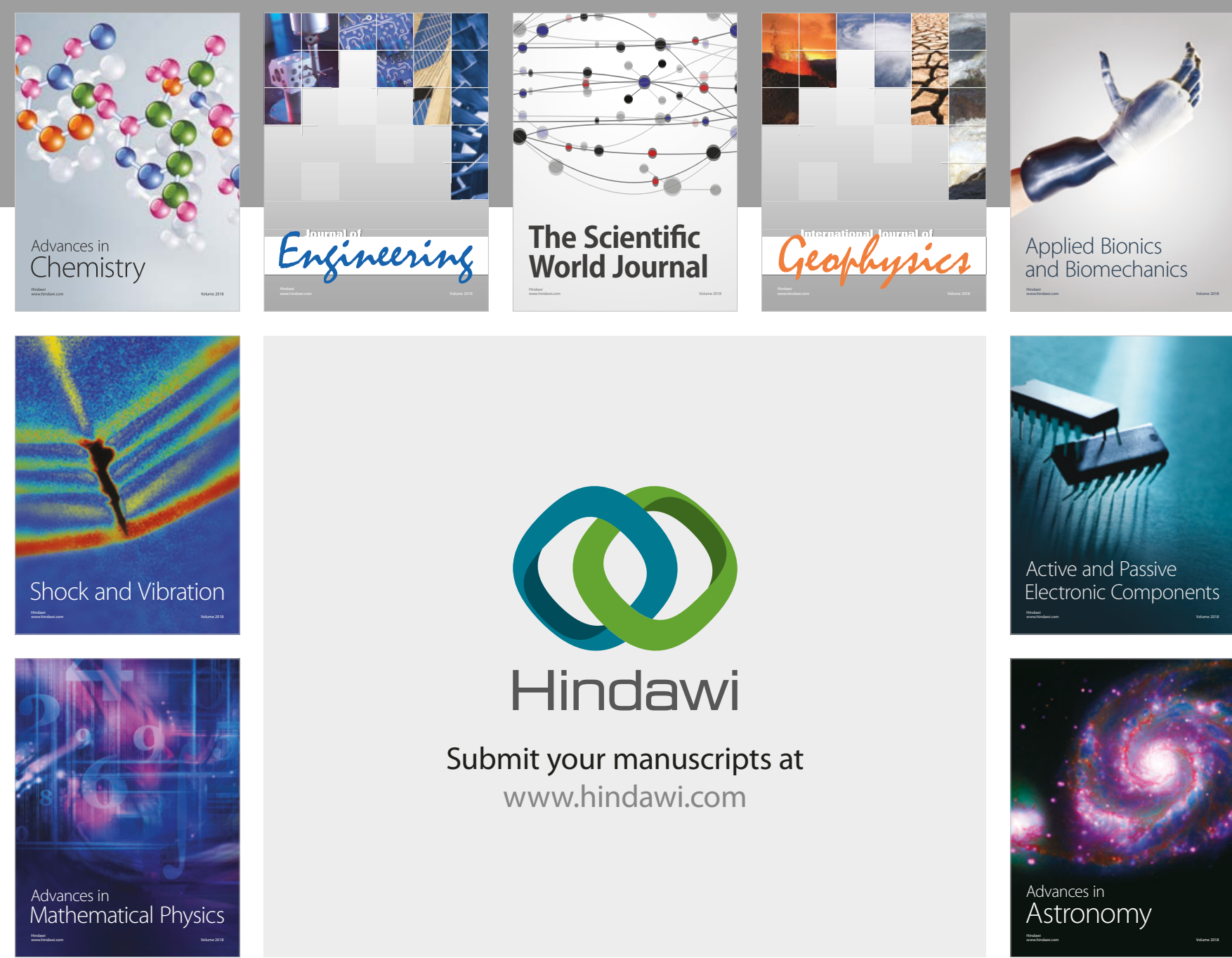

Submit your manuscripts at

www.hindawi.com

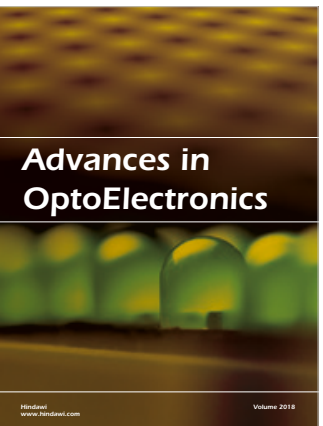

\section{Rotcting Machinery}
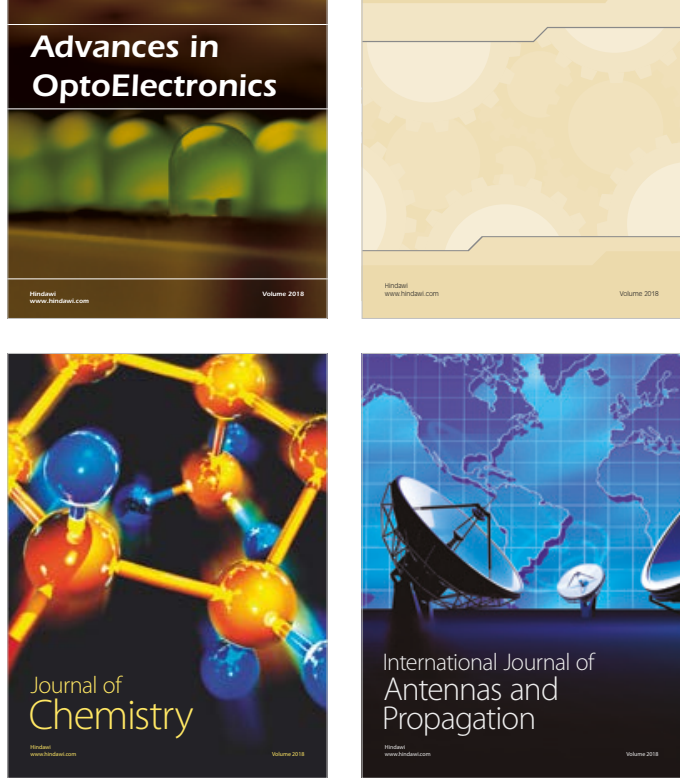

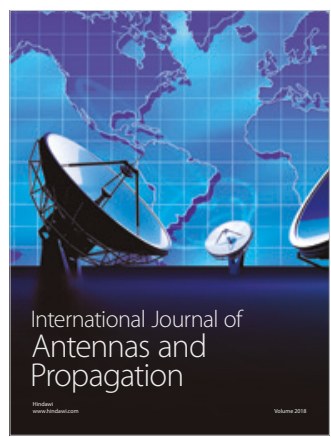

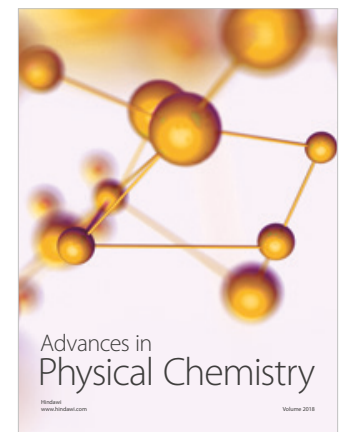

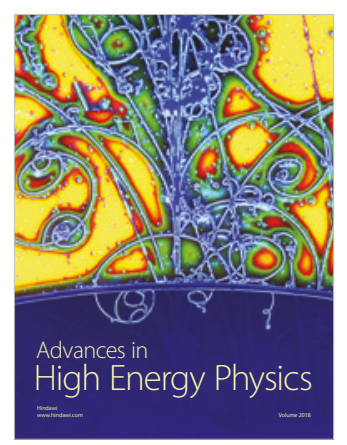

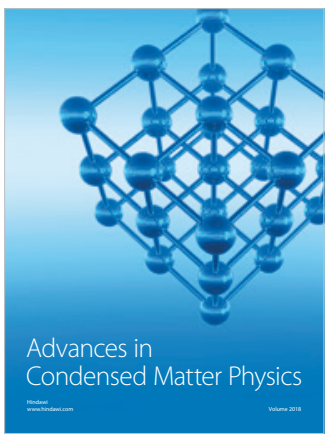

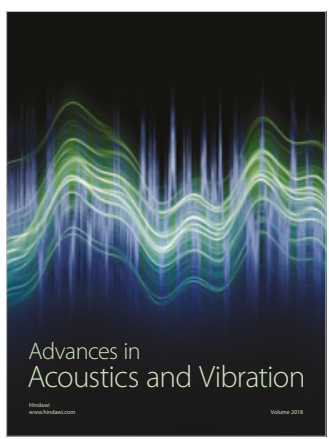

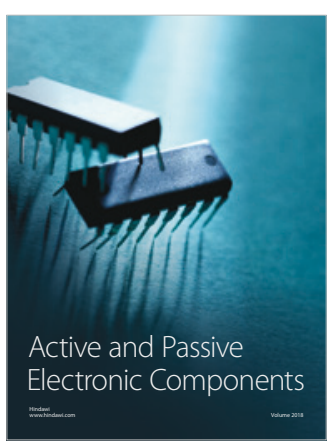
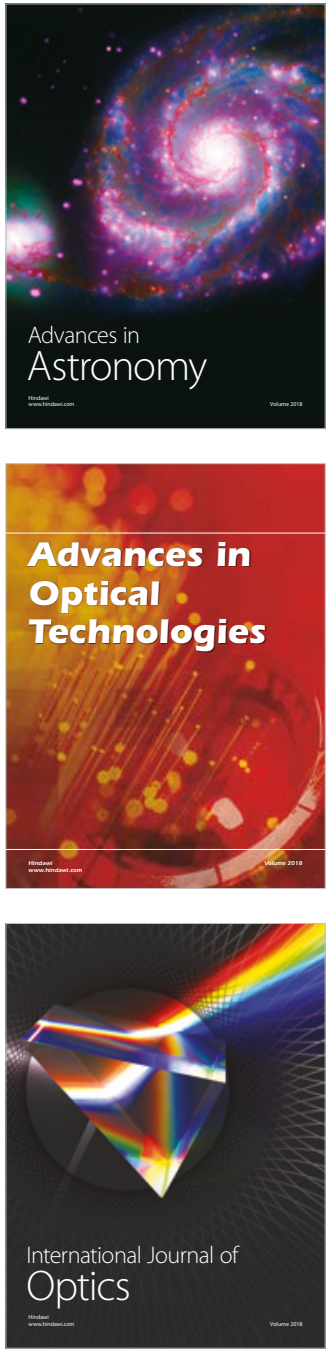